\title{
Outcomes of Magnetic Resonance Imaging Detected Occult Neck of Femur Fractures: Do They Represent a Less Severe Injury with Improved Outcomes?
}

\author{
Pradyumna Raval, MCh*', Alistair I.W. Mayne, MRCS*, Phey Ming Yeap, FRCR*, \\ Thomas Barry Oliver, FRCR ${ }^{\dagger}$, Arpit Jariwala, FRCS*, Sankar Sripada, FRCS* \\ Department of Orthopaedic and Trauma Surgery, Ninewells Hospital and Medical School, \\ University of Dundee, Dundee, Scotland, UK*, \\ Tayside Orthopaedic Rehabilitation Technology (TORT) Centre, Ninewells Hospital and Medical School, \\ University of Dundee, Dundee, Scotland, $U K^{+}$, \\ Department of Clinical Radiology, Ninewells Hospital and Medical School, Dundee, Scotland, UK
}

Purpose: Occult hip fractures in the elderly can be missed on standard radiographs and are a known cause of morbidity. These are generally diagnosed on either magnetic resonance imaging (MRI) or computed tomography scan, depending upon local hospital policy. While there is an abundance of literature on hip fractures in general, little is known about the clinical outcome of patients with occult hip fractures. The aim of this study was to review the demographics, injury characteristics, management and clinical outcome of patients diagnosed with occult femoral neck fractures on MRI.

Materials and Methods: Using an existing hospital database, a retrospective analysis of all patients with occult hip fractures diagnosed by MRI scan from 2005 to 2014 was conducted.

Results: Sixty-four patients (23 males and 41 females) were included. The mean duration of hospitalisation was 16 days. A significantly higher percentage of patients were discharged to their pre-existing residence compared to National Institute for Health and Care Excellence (NICE) commissioning guidelines (66\% vs. 45\%). The 30and 60-day mortalities were $3 \%$ and $10 \%$, respectively. Mortality was lower in patients who underwent internal fixation $(n=3 / 31)$ compared with those undergoing replacement (hemi/total hip arthroplasty) $(n=5 / 12)(P=0.056)$. Conclusion: Patients with occult hip fractures diagnosed on an MRI scan are more likely to be discharged to their pre-existing residence and have lower mortality rates compared to NICE guidelines and National Hip Fracture Database (NHFD).

Key Words: Hip fractures, Occult, Magnetic resonance imaging

Submitted: October 3, 2018 1st revision: December 26, 2018

Final acceptance: January 3, 2019

Address reprint request to

Pradyumna Raval, MCh

(https://orcid.org/0000-0002-8082-3458)

Department of Orthopaedic and Trauma Surgery, Tayside Orthopaedic Rehabilitation Technology (TORT) Centre, Ninewells Hospital and Medical School, University of Dundee, Dundee, DD1 9SY, Scotland, UK TEL: +44-7467472481 FAX: +44-1382-496322

E-mail: raval.prashant@igmail.com

This is an Open Access article distributed under the terms of the Creative Commons Attribution Non-Commercial License (http://creativecommons. org/licenses/by-nc/4.0) which permits unrestricted non-commercial use, distribution, and reproduction in any medium, provided the original work is properly cited.

\section{INTRODUCTION}

Proximal femoral fractures are a major cause of morbidity and mortality in the ever-increasing elderly population, and represent an increasing financial burden on healthcare systems ${ }^{1)}$. In the majority of patients presenting with a hip fracture, there is a clear fracture line on standard anteroposterior and/or lateral hip radiographs. However, in a small number of patients in which there is clinical suspicion but inconclusive radiographs, further imaging with a magnetic resonance imaging (MRI) scan is recommended. According to National Institute for Health 
and Clinical Excellence (NICE) guidelines, if MRI is contraindicated or not available within 24 hours, computed tomography (CT) scanning may be performed ${ }^{2}$.

Although there is an abundance of literature on hip fracture outcomes in general, much less is known about the outcomes of occult hip fractures diagnosed on $\mathrm{MRI}^{3-5)}$. Therefore, the purpose of the present study was to evaluate the demographics, injury characteristics, management and clinical outcome of patients diagnosed with occult femoral neck fractures on MRI.

\section{MATERIALS AND METHODS}

\section{Study Design}

This retrospective cohort study took place in a UK teaching hospital. Patients were identified using the existing Radiological Imaging Service (RIS) database; approval to access the database was granted by the local Caldicott Guardian. A total of 111 patients were diagnosed with an occult femoral neck fracture by an MRI scan between 2005 and 2014; however, after careful case-note review, only 64 of these patients met the inclusion criteria for the present study (Table 1). All patients with multiple fractures, pathological fractures and high energy injuries were excluded as this was thought to impact length of stay and mortality rates.

\section{Data Collection}

The hospital records of patients were reviewed and demographic characteristics (i.e., age, gender, side of injury [right/left]) were noted (Table 2). Radiological images were reviewed using the RIS. Other variables noted were the time from the initial hip radiograph to the MRI investigation, time from the MRI diagnosis to surgical management (in cases where surgery was performed), total duration of hospitalization, infection rates and mortality. Mortality was further sub-grouped as mortality within the first 30 days and within 90 days.

All MRI examinations were obtained on 1.5 Tesla scanners (Siemens, Erlangen, Germany). A standard trauma examination comprised of $5 \mathrm{~mm}$ short tau inversion recovery and $\mathrm{T} 1$ coronal series of the entire pelvis.

The decision for operative versus non-operative management and surgical procedure performed was chosen by the consultant in charge of the patient's care. Patients with inter-trochanteric neck femur fractures underwent internal fixation with a dynamic hip screw (DHS) while those with a trans-cervical neck femur fracture were treated with either internal fixation with a 2-hole DHS or arthroplasty. A note was made of the discharge destination of patients (i.e., to their preexisting residence or to a rehabilitation unit). Outcomes were compared to NICE guidelines and National Hip Fracture Database (NHFD).

\section{Statistical Analysis}

A statistical analysis was conducted using IBM SPSS software package version 21 (IBM Corp., Armonk, NY, USA). Student's $t$-tests were used to compare mean age and time from diagnosis to surgery. Pearson's chi-squared tests were used to compare the side of injury and final

Table 1. Patient Selection Criteria

\begin{tabular}{lc}
\hline \hline Inclusion criteria & Exclusion criteria \\
\hline Neck of femur and Intertrochanteric fractures & Sub-trochanteric fractures \\
Low impact injuries & High impact injuries \\
Isolated hip injuries & Multiple fractures \\
& Pathological fractures \\
\hline
\end{tabular}

Table 2. General Demographic Characteristic $(n=64)$

\begin{tabular}{lccc}
\hline \hline Variable & Male & Female & $P$-value \\
\hline Patient & $25(38.6)$ & $39(61.4)$ & $0.324^{*}$ \\
Age lyr) & $79.2 \pm 10.8$ & $81.9 \pm 9.0$ & $0.021^{+}$ \\
Side, right/left & $8 / 17$ & $24 / 15$ & $0^{-17}$ \\
\hline
\end{tabular}

Values are presented as number (\%), mean \pm standard deviation, or number only.

* Two sample Student $t$ test, ${ }^{+}$chi-square test. 
discharge destination. The statistical significance level was set at 0.05 for each significance test.

\section{RESULTS}

From 2005 to 2014, 64 patients (23 males and 41 females) were hospitalised with occult hip fractures diagnosed on an MRI scan. They were further subclassified according to their anatomical location (Fig. 1). There were 33 intracapsular hip fractures and 31 extracapsular fractures. The mean time from a negative hip radiograph to MRI investigation was three days (range, 1-5 days). Thirteen patients were managed nonoperatively, comprising of isolated greater trochanter and incomplete inter-trochanter fractures. Following MRI diagnosis, two-thirds of patients undergoing surgery had their procedure within 48 hours (mean, 2.06 days; range, 0-16 days). Fracture types, surgical procedures performed and mortality are detailed in Table 3 .

The mean duration of hospitalization was 16 days (range, 3-29 days). Two-thirds of patients returned to their pre-existing residence after being discharged, while one third went to rehabilitation centres. Two patients developed a post-operative superficial wound infection which was successfully treated with antibiotics. There

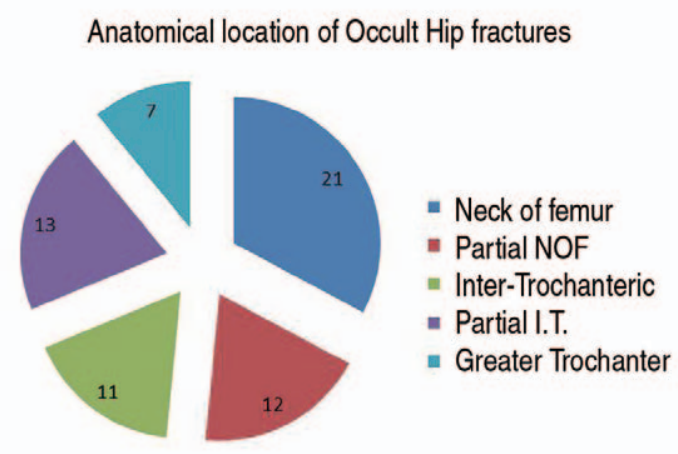

Fig. 1. Anatomical location of occult hip fractures. NOF: neck of femur fracture, I.T.: inter-trochanteric fractures. were no known cases of metalwork failure. Thirty- and ninety-day mortality rates were $3 \%$ and $10 \%$, respectively. The ninety-day mortality rates of intra-capsular versus extra-capsular fracture patients were not significantly different $(P=0.122)$. However, patients who underwent internal fixation had a lower mortality rate compared to those who underwent joint replacement procedures, although this was not statistically significant $(P=0.056)$.

\section{DISCUSSION}

The prevalence of occult hip fractures is approximately $3 \%{ }^{2}$. In the presence of clinical suspicion for hip fractures but negative radiographs, NICE guidelines recommend further imaging with an MRI scan. If MRI is contraindicated or not available within 24 hours, CT scanning may be performed ${ }^{2}$. MRI has been shown to detect occult fractures more accurately than CT scans and the $\mathrm{T} 1$ sequence is believed to be $100 \%$ sensitive in highlighting occult femoral neck fractures ${ }^{6-8}$. Occult hip fractures missed by 64 slice CT scan have been accurately detected by MRI'.

To our knowledge, this is the single largest study specifically reviewing outcomes of patients with occult hip fractures diagnosed on MRI scan. The results suggest that patients with occult neck femur fractures have a shorter length of stay, lower mortality rates and are more likely to be discharged to their pre-existing residence when compared with outcomes of the $\mathrm{NHFD}^{10)}$. The thirty- and ninety-day mortality rates were $3 \%$ and $10 \%$, respectively, values which compare favorably to results from the NHFD which reveal an overall thirty-day mortality rate of $6.7 \% \%^{10-12}$.

In our study, $66 \%$ of patients were discharged to their pre-existing residence, significantly better compared to NICE-commissioning guides where only $45 \%$ returned to their home ${ }^{13)}$. The improved outcome seen in patients who sustain occult neck of femur fractures may be because these injuries represent lower energy injuries

Table 3. Type of Fracture, Surgical Procedure and Mortality

\begin{tabular}{lccc}
\hline \hline Variable & Alive & Dead & $P$-value \\
\hline Intra-capsular fracture & 25 & 8 & $0.122^{*}$ \\
Extra-capsular fracture & 28 & 3 & $0.056^{*}$ \\
Joint replacement & 12 & 5 & 3 \\
Internal fixation (DHS) & 31 & 3 & \\
\hline
\end{tabular}

DHS: dynamic hip screw.

* Chi-square test. 


\section{Hip \& Pelvis}

Pradyumna Raval et al. Outcomes of MRI-detected Occult Neck of Femur Fractures

compared to those with displaced fractures. Therefore the associated morbidity is lower compared to patients sustaining a displaced neck femur fracture where more significant tissue disruption and internal hemorrhage probably occurs with the primary injury. Similarly, in those managed with internal fixation, the surgical procedure is technically less challenging compared to patients with a displaced fracture where fracture reduction must be achieved before a definitive fixation. These factors are likely to explain the reduced length of stay, lower mortality rates and higher discharge to preinjury residence in patients with occult hip fractures.

MRI investigation can be very useful in diagnosing incomplete inter-trochanteric fracture, as illustrated in Fig. 2 and 3. There is some debate about the need to fix incomplete inter-trochanteric fractures. In a review of eight patients with occult inter-trochanteric fractures diagnosed by MRI, Alam et al. ${ }^{3}$ reported successful outcomes for five patients managed non-operatively. However a recent systematic review by Kim et al. ${ }^{14)}$ reported that of the 61 incomplete inter-trochanteric fractures treated with surgical fixation, no complications were reported and they concluded that due to the risks of incomplete non-displaced fractures progressing to complete displaced fractures, conservative management should be undertaken with caution. Given that the aim of treating hip fractures is to enable early mobilization, we would also advocate for early fixation of occult intertrochanteric hip fractures.

Patients treated with internal fixation had a lower mortality rate compared to those who underwent joint

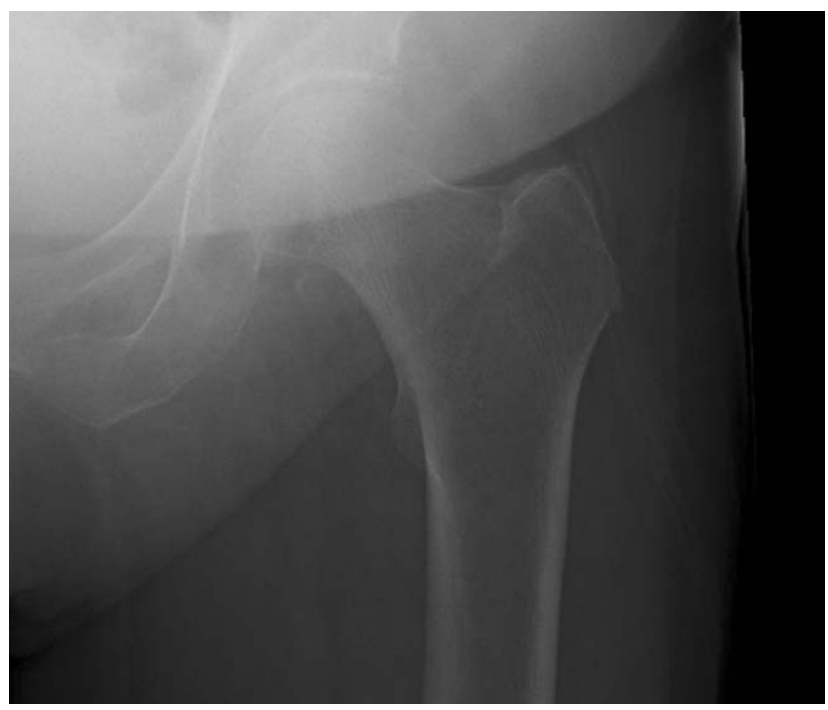

Fig. 2. Normal left hip radiograph. replacement procedures $(P=0.056)$. This may be because sliding hip screw fixation in an undisplaced hip fracture is a less invasive procedure compared to a replacement procedure.

Pihlajamäki et al. ${ }^{5)}$ reported on the long-term outcomes of 66 conservatively managed undisplaced femoral neck fractures followed up for a mean of 18.3 years. Although they reported favorable outcomes with conservative management with no cases of fracture displacement or avascular necrosis, these were fatigue fractures of the femoral neck in young military recruits and so represent a different injury compared with occult femoral neck fractures seen in the elderly.

Beloosesky et al. ${ }^{15)}$ reported on a smaller cohort of 29 patients with occult hip fractures diagnosed on bone scan and compared the results to a randomly chosen

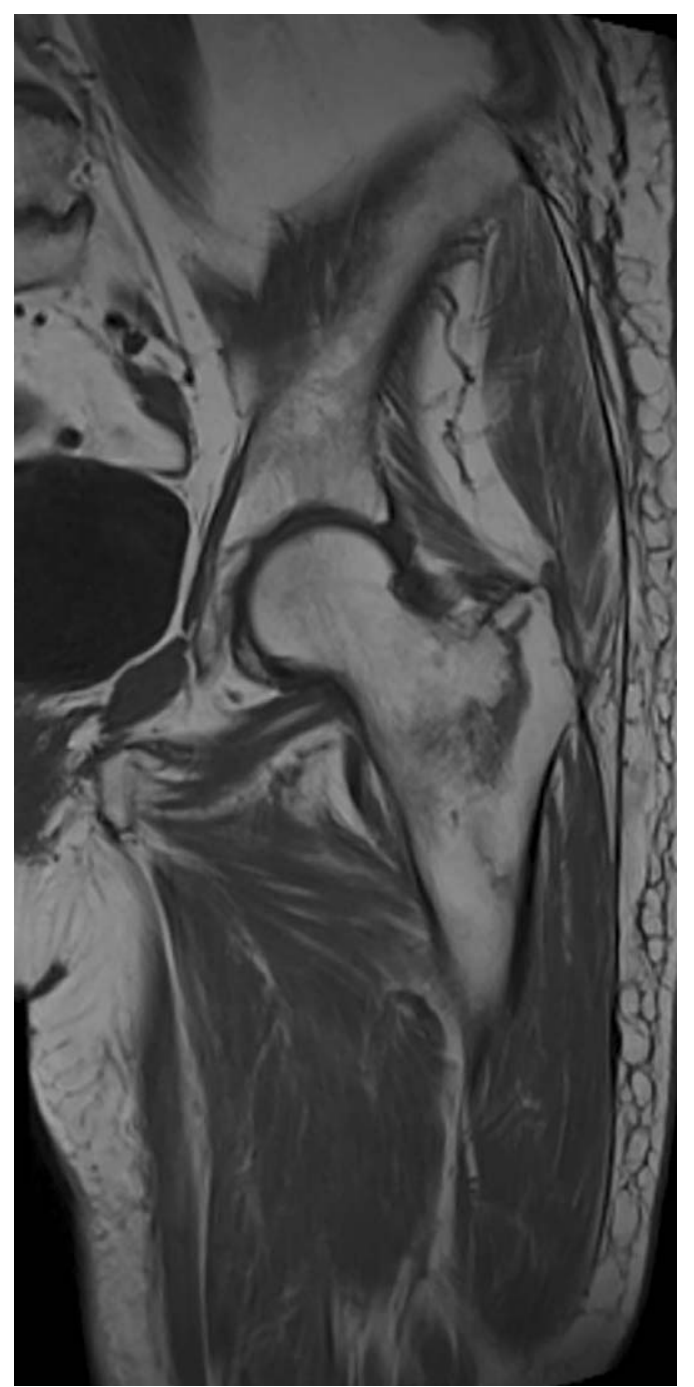

Fig. 3. Incomplete inter-trochanteric fracture diagnosed on magnetic resonance imaging. 
group of 94 patients diagnosed with a hip fracture by plain radiographs. In this study, those with hip fractures diagnosed on plain radiographs had a significantly increased rate of complications. There was no significant difference in inpatient length of stay or 30day mortality between groups. The authors also reported that there were no significant differences in survival between the groups during 13 years of follow-up.

Our study has certain limitations. We were unable to collect detailed information about the body mass index, or American Society of Anesthesiologists (ASA) score for our patients. Due to these limitations, a multivariate analysis could not be performed; however, we performed a univariate analysis on the available data. All of our patients were ambulant at the time of injury and were mobilized before discharge to their usual residence. This was our primary clinical outcome of interest; however, we did not quantify it by performing any outcome measure scoring.

\section{CONCLUSION}

Occult hip fractures represent a unique subset of proximal femoral fractures. This study has shown a reduced length of stay, reduced need for rehabilitation following discharge and reduced short-term mortality compared to national data. This injury-specific information may help facilitate a more informed discussion with patients sustaining occult hip fractures.

\section{CONFLICT OF INTEREST}

The authors declare that there is no potential conflict of interest relevant to this article.

\section{REFERENCES}

1. British Orthopaedic Association. The care of patients with fragility fracture. London: British Orthopaedic Association; 2007.
2. National Clinical Guideline Centre. NICE Clinical Guidelines, No. 124. The management of hip fracture in adults. London: Royal College of Physicians (UK); 2011.

3. Alam A, Willett K, Ostlere S. The MRI diagnosis and management of incomplete intertrochanteric fractures of the femur. J Bone Joint Surg Br. 2005;87:1253-5.

4. Chana R, Noorani A, Ashwood N, Chatterji U, Healy J, Baird P. The role of MRI in the diagnosis of proximal femoral fractures in the elderly. Injury. 2006;37:185-9.

5.Pihlajamäki HK, Ruohola JP, Weckström M, Kiuru MJ, Visuri TI. Long-term outcome of undisplaced fatigue fractures of the femoral neck in young male adults. J Bone Joint Surg Br. 2006;88:1574-9.

6. Iwata T, Nozawa S, Dohjima T, et al. The value of T1weighted coronal MRI scans in diagnosing occult fracture of the hip. J Bone Joint Surg Br. 2012;94:969-73.

7. Lubovsky O, Liebergall M, Mattan Y, Weil Y, Mosheiff R. Early diagnosis of occult hip fractures MRI versus CT scan. Injury. 2005;36:788-92.

8. Hossain M, Barwick C, Sinha AK, Andrew JG. Is magnetic resonance imaging (MRI) necessary to exclude occult hip fracture? Injury. 2007;38:1204-8.

9. Hakkarinen DK, Banh KV, Hendey GW. Magnetic resonance imaging identifies occult hip fractures missed by 64-slice computed tomography. J Emerg Med. 2012;43: 303-7.

10. Royal College of Physicians. National Hip Fracture Database (NHFD) annual report 2017. London: Royal College of Physicians; 2017.

11. Todd CJ, Freeman CJ, Camilleri-Ferrante C, et al. Differences in mortality after fracture of hip: the east Anglian audit. BMJ. 1995;310:904-8.

12. Hapuarachchi KS, Ahluwalia RS, Bowditch MG. Neck of femur fractures in the over 90s: a select group of patients who require prompt surgical intervention for optimal results. J Orthop Traumatol. 2014;15:13-9.

13. National Institute for Health and Clinical Excellence (NICE). NICE Guidance CMG 46. The management of hip fractures in adults. London: NICE; 2012.

14. Kim SJ, Ahn J, Kim HK, Kim JH. Is magnetic resonance imaging necessary in isolated greater trochanter fracture? A systemic review and pooled analysis. BMC Musculoskelet Disord. 2015;16:395.

15. Beloosesky Y, Hershkovitz A, Guz A, Golan H, Salai M, Weiss A. Clinical characteristics and long-term mortality of occult hip fracture elderly patients. Injury. 2010;41: 343-7. 